\title{
An interval scale of saturation for the pigeon
}

\author{
BRUCE A. SCHNEIDER \\ University of Toronto, Mississauga, Ontario L5L 1C6, Canada
}

\begin{abstract}
Pigeons were required to discriminate between "identical" vs. "different" pairs of lights in a yes/no signal-detection task with a symmetrical payoff matrix. If the two lights projected on the two halves of the bipartite field constituting the center response key in a three-key chamber were identical in wavelength composition, then a single peck on the left key was reinforced with food. If the two lights differed in wavelength composition, then right-key pecks were reinforced. Each pigeon experienced all possible pairs (55) of 11 lights having the same dominant hue $(630 \mathrm{~nm})$ but differing in colorimetric purity. The percentage of correct responses was taken as a measure of the dissimilarity between the two lights constituting a pair. The rank-order information available in these dissimilarity measures was used to determine an interval scale of saturation in the pigeon. Saturation was found to be linearly related to colorimetric purity.
\end{abstract}

Phenomenologically, colors are described with respect to the attributes of hue, saturation, and brightness. While hue and brightness have been the focus of many studies of color vision in pigeons (see Donovan, 1978 , for a review), only two studies have attempted to measure saturation (the perceived chromatic content of a light or surface relative to an achromatic light or surface). Blough (1975) used generalization procedures in an attempt to determine the relative saturation of spectral lights. She assumed that the spectral lights most similar to white light, that is, those with the least degree of saturation, should elicit more responses in the generalization test than the spectral lights which are more saturated and, therefore, appear to differ more from white. In two experiments, Blough found the spectral region between 550 and $600 \mathrm{~nm}$ to be the least saturated portion of the spectrum in the sense that there was a greater degree of generalization between a white light (color temperature $=3,050^{\circ} \mathrm{K}$ ) and spectral lights in this region than on either side of it.

Romeskie and Yager (1976a, 1976b) inferred the relative saturation of monochromatic lights from measurements of the spectral photochromatic interval for the pigeon. The spectral photochromatic interval is the difference in intensity levels (expressed in log units) between a monochromatic light at absolute threshold and the intensity level at which it can be distinguished from an equally bright white light. Graham and Hsia (1969) have shown that for humans, wavelengths that appear strongly saturated have small photochromatic intervals, and those that appear weakly saturated have large photochromatic intervals. Hence, photochromatic interval can be used as an index of

This research was supported by a grant from the Natural Sciences and Engineering Research Council of Canada. Reprints may be obtained from Bruce Schneider, Department of Psychology, Erindale College, University of Toronto, Mississauga, Ontario LSL 1C6, Canada. saturation. Romeskie and Yager (1967b) found that photochromatic intervals were larger around 600$620 \mathrm{~nm}$. If the photochromatic interval size is inversely related to saturation, this, of course, implies that the wavelengths between 600 and $620 \mathrm{~nm}$ are the least saturated. The discrepancy between Blough (1975) and Romeskie and Yager (1976b) may be due to the fact that the correlated color temperature of the white light employed by Romeskie and Yager was $4,800^{\circ} \mathrm{K}$ as opposed to the $3,050^{\circ} \mathrm{K}$ white light employed by Blough. Another possibility, however, is that the relationship between spectral photochromatic interval and saturation shown to exist for humans might not hold for pigeons.

The techniques used in the above studies were employed to measure the relative saturation of spectral lights. However, both of these approaches cannot determine how saturation changes as a function of colorimetric purity for any given wavelength of light. Colorimetric purity is defined as the proportion of spectral light in a mixture of white light and a pure spectral light. Thus, if we mix a monochromatic light of wavelength $630 \mathrm{~nm}$ (human red) with a white light, the apparent saturation of the mixture will be some function of its colorimetric purity. For colorimetric purities near zero (a high proportion of white light to 630-nm light), the mixture will appear to be a highly desaturated red, while for colorimetric purities close to 1.0, the mixture will appear highly saturated (close to a pure red). Several studies involving humans as subjects have determined how the apparent saturation of a light varies as a function of its colorimetric purity (e.g., Indow, 1967; Indow \& Stevens, 1966; Panek \& Stevens, 1966). The goal of the present study is to determine for pigeons how the apparent saturation of a. red light varies as a function of its colorimetric purity.

If perceived saturation is a unidimensional psychological experience, then the saturation of a light can be 
represented as a point on a line segment where the distances between points correspond to the perceived differences in saturation among the stimuli. In the present paper, a saturation scale is derived for pigeons from measures of the psychological differences between lights varying only in colorimetric purity. Measures of the psychological difference or "distance" between lights were obtained from the pigeon using a yes/no signal-detection technique. Pigeons were required to discriminate between "identical" vs. "different" pairs of lights which varied only in colorimetric purity. The accuracy of this discrimination with respect to a given pair of lights was taken as a measure of the perceived saturation difference between them. It is assumed that if the difference in perceived saturation is greater for light pair $(x, y)$ than for pair $(w, z)$, then the accuracy of discrimination for pair $(x, y)$ will be greater than that for pair $(w, z)$. Thus, we assume that discrimination accuracy is monotonic with saturation difference.

\section{METHOD}

\section{Subjects}

The subjects were six White Carneaux pigeons from the Palmetto Pigeon Plant, Sumter, South Carolina. They were tested 5 days a week and fed enough grain after each session to be maintained at $85 \%$ of their ad-lib weights. A vitamin supplement was added to their water once a week.

\section{Apparatus}

Experimental chamber. The pigeon's compartment $(31 \times 31 \times$ $32 \mathrm{~cm}$ ) was constructed of plywood on five sides and had a wiremesh grid on the floor. An aluminum panel separated the subject's compartment from the stimulus and food delivery devices. Three Lehigh Valley response keys were centered behind three $2.54-\mathrm{cm}$ holes in this panel. All three response keys were positioned $22 \mathrm{~cm}$ above the floor of the chamber. The middle response key was centered on the panel, and the two side keys were displaced $7.5 \mathrm{~cm}$ from the center of the panel. Each side key could be illuminated by a G.E. $182928-\mathrm{V}$ bulb filtered through a Wratten $80 \mathrm{~B}$ filter and Wratten neutral density filters to shift its color temperature above $4,000^{\circ} \mathrm{K}$ and adjust its luminance to 9.2 nits at its brightest point as measured by an S.E.I. photometer.'

Located $16 \mathrm{~cm}$ below the center key was a $6.2 \times 5.2 \mathrm{~cm}$ opening in the panel through which a hopper filled with mixed grain could be made available. The reinforcer was $3-\mathrm{sec}$ access to grain. During the reinforcement cycle, the hopper was illuminated by a G.E. 1829 bulb also filtered by a Wratten $80 \mathrm{~B}$ filter.

Seven G.E. 1829 bulbs, mounted behind an $80 \mathrm{~B}$ filter and a $12 \times 11.5 \mathrm{~cm}$ piece of ground glass on the ceiling of the chamber, were used to maintain a constant state of adaptation. The average luminance of the adapting light as measured by an S.E.1. photometer was 58.9 nits. The average luminance of the chamber walls, which were painted flat black, was .1 nits. The average luminance of the unpainted sections of the metalic front panel was .6 nits. The adaptation light remained on for the entire session except for the presentation of the test signal.

Optical system. A bipartite field $(1.7 \mathrm{~cm}$ diam) was projected directly on the center key by the projection system shown in Figure 1. The two light sources (0) were voltage-regulated $500-\mathrm{W}$ Tungsten-Halogen lamps with a color temperature of $3,000^{\circ} \mathrm{K}$. One lamp provided the source for the 630-nm light, and the other provided the source for the "white" light. Two beams of light were taken from each source to form the separate halves of the bipartite stimulus. In each channel, light from the source passed

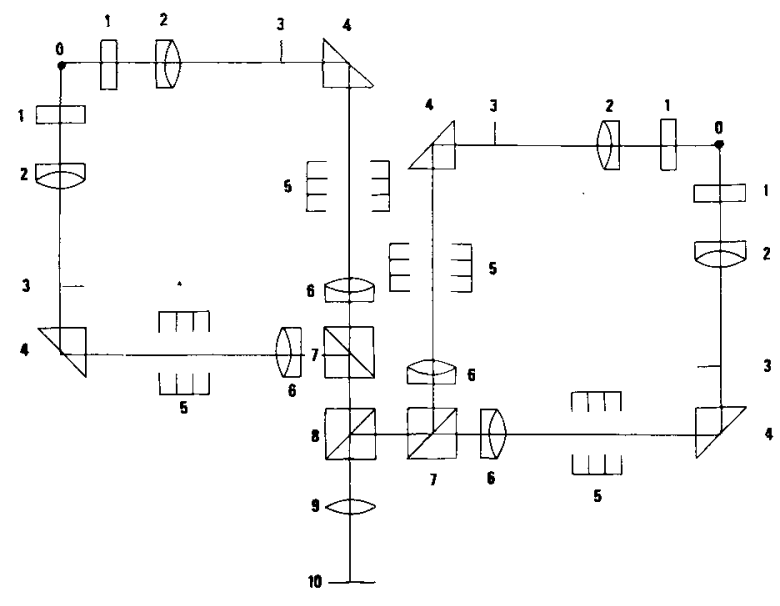

Figure 1. Schematic of the optical system employed in Experiments 1 and 2 .

through heat-absorbing glass (1) and a collimating lens (2). A field stop (3) was inserted at this point to block one half of the beam to form the two halves of the bipartite field. After being reflected off a right-angle prism (4), the collimated light passed through a filter box (5) containing blocking filters, neutral-density filters, and, in the case of the monochromatic channels, interference filters. After passing through the second lens (6), the beams from the two channels from the same light source were combined by means of a beam splitter (7). A second beam splitter (8) united the beams from the 630-nm and white channels. A final lens (9) was used to complete the projection system and image the edge of the field stop (3) on the ground-glass screen (10) that constituted the center response key. An opaque black line divided the response key down the center (width $=1 \mathrm{~mm}$ ). Each edge of the bipartite field was focused on this black line. Thus, two homogeneous fields of light separated by $1 \mathrm{~mm}$ appeared on the center key.

Motors attached to the filter boxes interposed the neutral density filters programmed for a particular trial. Matched interference filters (Bauch and Lomb, No. 44, peak wavelengths $=630$ and $633 \mathrm{~nm}$ ) were employed to provide the monochromatic fields. Since these filters pass three modes of energy, one in the ultraviolet, one in the visible (the nominal value of the filter), and one in the infrared, a No. 15 Kodak blocking filter was used to eliminate the short-wavelength mode. The heat-absorbing glass eliminated the infrared mode of energy. The color temperature of the light in the achromatic field was adjusted upward by inserting a Kodak Wratten $80 \mathrm{~B}$ filter in the achromatic optical channels. A Kodak Wratten No. 2A filter was also inserted in the white-light channels to eliminate the ultraviolet portion of the spectrum (see Wright, 1972). The intensities of the lights in all four channels were controlled by inserting Ealing neutral-density filters in the optical channels by means of the motor-driven filter changer. To obtain intensity steps smaller than $.1 \log$ unit, glass cover slips were also interposed in the optical channels along with the neutral-density filters.

Stimulus calibrations. The spectral transmittances of the heatabsorbing glasses, the interference filters, the Kodak Wratten $2 \mathrm{~A}$, 15 , and $80 \mathrm{~B}$ filters, and the interference filters were determined by using a Unicam SP 1800 spectrophotometer. The correlated color temperature of the "white light" employed in this experiment was computed in the following manner. First, the spectral radiance of a black body radiator with a color temperature of $3,000^{\circ} \mathrm{K}$ (the color temperature of the bulb) was adjusted by the transmittances of the nonneutral optical components in the channel (the heat-absorbing glass, and the Kodak Wratten $2 \mathrm{~A}$ and $80 \mathrm{~B}$ filters). Second, the correlated color temperature was computed from the 
adjusted spectrum. The correlated color temperature of the white light measured in this way was $5,400^{\circ} \mathrm{K}$ with chromaticity, coordinates $x=.335, y=.370$. The luminance of this white stimulus was 58.9 nits as measured by an S.E.I. photometer.

With the chromatic channels blocked so that only white light was projected on the two halves of the split field, neutral density filters and cover slips were used to equalize the luminance of both halves of the split field. The luminance of this white-light stimulus was adjusted to 58.9 nits as measured by an S.E.1. photometer. To equate the white light and the 630-nm light in brightness, the following procedure was employed. First, an interference filter with a peak wavelength of $590 \mathrm{~nm}$ was placed in the chromatic channel projecting onto the left half of the field, while the white light stimulus appeared in the right half of the split field. The luminance of the $590-\mathrm{nm}$ stimulus was adjusted by neutral-density filters until it matched the luminance of the white field (as measured by an S.E.1. photometer). In this fashion, the luminance of the $590-\mathrm{nm}$ stimulus was equated with the luminance of the white-light stimulus as perceived by the human eye. It can be shown that when the radiant intensity of a $590-\mathrm{nm}$ light and the radiant intensity of a white light with a color temperature of $5,400^{\circ} \mathrm{K}$ are adjusted to produce equal human luminance, they will be equally bright to the pigeon as well. The logic of this argument is as follows. If the two lights are equal in brightness for a human, then

$$
P_{590} V_{590}=\int P_{\lambda} V_{\lambda} d \lambda,
$$

where $P_{590}$ is the radiant flux of the $590-\mathrm{nm}$ stimulus, $V_{590}$ is the value of the luminosity coefficient at $590 \mathrm{~nm}, P_{\lambda}$ is the radiant intensity of the white-light source at wavelength $\lambda$, and $V_{\lambda}$ is the value of the luminosity coefficient at that wavelength. If the same intensities of the 590 -nm light and white light which produced equality in brightness in humans are now multiplied by the luminosity coefficients determined by Blough (1957) for pigeons, the equality still holds within $.015 \mathrm{log}$ units. ${ }^{2}$ Hence, equating a $590-\mathrm{nm}$ light and a white light in terms of human brightness also equates them in terms of pigeon brightness. Once this brightness match was made, the radiant intensity of the $590-\mathrm{nm}$ light was measured using a United Detector Technology $40 \mathrm{X}$ radioneter. The radiant intensity necessary to produce an equally bright light for pigeons at $630 \mathrm{~nm}$ was determined using the pigeon luminosity coefficients determined by Blough (1957). Neutral-density filters were then inserted into the chromatic channels to produce this radiant intensity at $630 \mathrm{~nm}$. In this fashion, the $630-\mathrm{nm}$ stimulus and the white light were equated for pigeon brightness.

With both chromatic channels blocked, matched white stimuli were produced on both halves of the split field. With both whitelight channels blocked, matched 630-nm stimuli appeared on both halves of the split field. To produce a light of intermediate colorimetric purity on, say, the left side of the split field, the chromatic channel projecting to the left side was added to the achromatic channel projecting to the same side. This mixture produced a colorimetric purity intermediate between 0 and 1 , the exact value depending on the relative amount of attenuation added into the chromatic and achromatic channels. To maintain constant pigeon brightness at all colorimetric purities, attenuation values $x$ and $y$ were constrained to sum to 1.0 so that $\mathrm{xL}_{630}+y L_{w}=L_{w}$, where $\mathrm{L}_{630}$ and $\mathrm{L}_{w}$ refer to the pigeon luminances of the unattenuated $630-\mathrm{nm}$ and white lights, respectively. (Combinations of neutraldensity filters and cover slips provided the attenuation in the two channels. Because it was not always physically possible to select a combination in which the attenuation values in both channels summed exactly to 1.0 , constant pigeon brightness was maintained only to within $.04 \mathrm{log}$ units.) Twelve colorimetric purities were produced in this fashion. Their values were: $0.0, .06, .16, .24, .32$, $.44, .56, .68, .76, .84, .94$, and 1.0 .

\section{Procedure}

Final procedure. At the beginning of a trial, the bipartite field was projected on the center key. The side keys were dark. A single response on the center key (an "observing" response) illuminated the side keys. If the two halves of the center key were identical in wavelength composition, then a response to the left side key was designated as correct. If the two halves of the field differed in wavelength composition, then a response on the right side key was designated as correct. Three-second access to food followed $20 \%$ of the correct (right or left) responses. The other $80 \%$ were followed by $.25 \mathrm{sec}$ access to food. This latter time period was too brief for the pigeon to be able to eat any grain. It simply served as "feedback" for correct responses. Incorrect responses (responding left for different and right for identical wavelength compositions) produced an intertrial period. Time between a side key response and the beginning of the next trial was $7.5 \mathrm{sec}$. Keys were extinguished when a response occurred to a lighted side key. The adaptation light was turned on during the intertrial period. Each session consisted of 240 trials.

The procedure is equivalent to a yes/no signal-detection paradigm with a symmetrical payoff matrix. Only a single pair of stimuli was employed per daily session. The four possible stimulus combinations appeared during a session with equal frequency: (1) stimulus $i$ on both halves of the field, (2) stimulus $j$ on both halves of the field, (3) stimulus $i$ on the left and stimulus $j$ on the right, and (4) stimulus $j$ on the left and stimulus $i$ on the right. Since the payoff matrix was constant throughout Experiments 1 and 2, response bias should have remained constant. Thus, the percentage of correct responses should be monotonically related to d' or any other measure of discriminability.

In Experiment 1, each pigeon experienced all possible pairs (55) of 11 stimuli differing in colorimetric purity $(0.0,06, .16, .24$, $.32, .44, .56, .68, .76, .84$, and .94$)$. Only one pair was presented per daily session. Each bird was presented these 55 pairs in a different random order. Sessions were conducted 5 days a week. Experiment 2 was a simple replication of Experiment 1, using different random orders for the presentation of the stimuli.

Preliminary training. Training procedures were similar to those employed by Schneider (1972). Initially, 10 birds were trained. The best 6 out of 10 birds were chosen for the final experiments. Selection was based on performance accuracy and stability. In particular, those birds with the highest performance accuracy were chosen as long as they consistently completed sessions. Prior to the beginning of Experiment 1, each bird had experienced over 100 sessions with various pairs of $630-\mathrm{nm}$ lights varying in colorimetric purity. Hence, each bird had considerable experience at this task.

\section{RESULTS}

In Experiment 1, each of six pigeons experienced every possible pair of 11 stimuli (630-nm lights) varying in colorimetric purity ( 55 pairs). The accuracy of discrimination for a pair (number of correct choices divided by number of trials) was taken as a measure of the dissimilarity of the two stimuli comprising that pair. A rank was then assigned to each of the 55 stimulus pairs based on these accuracy measures for each pigeon. These ranks represent an ordinal scale of perceived color difference for the pigeon for $630-\mathrm{nm}$ lights varying in colorimetric purity. Kendall's coefficient of concordance $(W)$ was used to determine the extent of the agreement among the rankings for the six pigeons. The coefficient of concordance was .85 in Experiment 1 and .86 in Experiment 2, indicating good agreement across subjects.

These ranks were then averaged across subjects to obtain a rank ordering of color difference for each experiment. The Spearman rank-order correlation 
coefficient between the average rank for Experiment 1 vs. the average rank for Experiment 2 was .965 . Hence, Experiment 2, may be considered as a reasonable replication of Experiment 1.

If saturation is a unidimensional sensory experience, then we should be able to represent the saturation of each stimulus as a point on a line segment, on which the distance between points is monotonic with the rank order of color difference obtained in Experiments 1 and 2 . Accordingly, the ranks were averaged across the two experiments to arrive at a representative rank order of color difference for the 55 pairs of $630-\mathrm{nm}$ lights that differed in colorimetric purity. This average rank order served as input to a nonmetric scaling program (Carvellas \& Schneider, 1972) which determined the best one-dimensional representation for the 11 stimuli. Stress (Kruskal, 1964) for this configuration was $6 \%$.

To evaluate how closely this one-dimensional representation approximated an interval scale of saturation, the index of metric determinacy $(M)$ was estimated from Young's (1970) nomogram. $M$ is the squared Pearson correlation coefficient between the true distances (whose rank ordering serves as the input to the algorithm) and the distances produced by the algorithm. Hence, $M$ varies between 0 and 1 , and $M=1$ means that the true distances have been perfectly reconstructed. In no empirical investigation using these techniques are the true distances known, but Young (1970) provided a nomogram for estimating $\mathbf{M}$ from the number of points, number of dimensions, and stress-all of which are available. The result is that, if $\mathrm{M}$ is sufficiently high (above .98, say), the point coordinates produced by the algorithm are properly regarded as an interval scale representation of the original points. In the present case, $M$ was conservatively estimated as .98 . Hence, the projection values obtained from the program may be taken as an interval scale of saturation for the pigeon.

Figure 2 plots the saturation values as a function

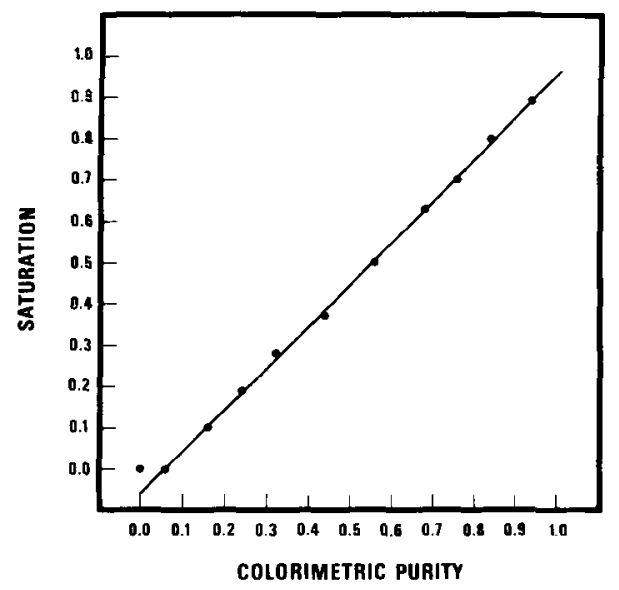

Figure 2. Saturation values as a function of colorimetric purity for the pigeon.

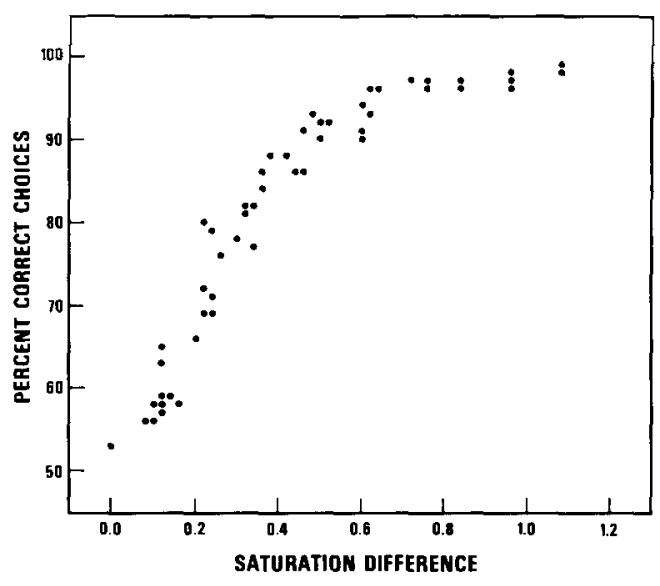

Figure 3. Discrimination accuracy as a function of saturation difference.

of colorimetric purity. A linear function describes the data quite well for colorimetric purities between .06 and .94 (the straight line was fit by the method of least squares to the highest 10 points). The data also suggest, the presence of a threshold for the detection of hue since the saturation values for colorimetric purities 0.0 and .06 are identical.

Figure 3 shows the relationship between discrimination accuracy (averaged across birds and experiments) and saturation difference. A one-dimensional nonmetric analysis attempts to assign scale values to the stimuli such that differences among these scale values are monotonically related to the measure of dissimilarity (discrimination accuracy in the present case). The negatively accelerated function in Figure 3 shows that the nonmetric scaling routine was reasonably successful in the sense that the relationship between discrimination accuracy and saturation difference is indeed monotonic.

\section{DISCUSSION}

Previous scaling techniques employed in animal psychophysics have required fairly strong assumptions concerning the relationship between behavioral measures and sensory attributes for scale construction. For example, Herrnstein and Van Sommers (1962) trained pigeons to respond at different rates to lights of different intensities. The response rates at these training intensities were constrained in such a way that response rate was a power function of intensity. When testing at intermediate intensities, Herrnstein and Van Sommers found that the intermediate stimuli elicited intermediate response rates which were consistent with a power law formulation. However, as Blough (1965) pointed out, the power law was, in a very direct sense, "built into"' the response measure. Even if a particular function were not built into the response measure, it would still be necessary to assume 
that response rate was proportional to sensory magnitude in order to determine the form of the psychophysical function. Given the number of factors that are known to influence response rate, it seems unreasonable to assume anything more than a monotonic relationship between response rate and magnitude.

Boakes (1969) employed a bisection technique to scale brightness. In this procedure, pigeons were trained to peck right for a bright stimulus and left for a dim one. Intermediate stimuli were then presented to determine the stimulus which produced equal pecking on both keys. Presumably, this stimulus bisected the sensory interval. However, as Blough and Blough (1977) point out, response factors, such as position preference, will alter the probability of a side-key response to a particular stimulus. Hence, response factors may very well bias the location of the bisection point. Since there is no easy way to control or compensate for such potential response biases in the bisection procedure, they create a problem for the use of bisection techniques in animal psychophysics.

The present technique is less sensitive to these response factors. Since the payoff matrix was constant throughout Experiments 1 and 2, response bias should remain constant. Thus, the percentage of correct responses should be monotonically related to $\mathrm{d}^{\prime}$, or other measures of discriminability. An additional advantage of the present technique is that it requires a fairly weak assumption for the construction of an interval scale of sensation-namely, that discrimination accuracy be monotonic with perceived differences in colorimetric purity. Thus, metric assumptions relating response measures, such as response rate, to psychological magnitude are circumvented.

\section{Comparisons with Human Data}

The low value of stress and the high value of $M$ indicate that we may properly regard the scale values obtained here as constituting interval scale measurement of saturation for the pigeon. Figure 2 indicates that saturation is linear with colorimetric purity for the pigeon. Human data for the saturation of a $630-\mathrm{nm}$ light obtained by the method of equisection also yield a scale of saturation that is linear with colorimetric purity (Indow, 1967). A similar finding has also been reported for other wavelengths (Indow \& Stevens, 1966). In an equisection task, subjects are asked to adjust stimuli to produce equal differences in saturation. In our present technique, we assume that discrimination accuracy is monotonic with saturation difference. Hence, when sensory differences are scaled in both pigeons and humans for the saturation of a $630-\mathrm{nm}$ light, the result is a scale of saturation which is linear with colorimetric purity.

However, with human subjects, saturation scales, constructed using magnitude or ratio estimation techniques, do not yield scales of saturation which are linear with colorimetric purity. Indow (1967), Indow and Stevens (1966), and Panek \& Stevens (1966) all found that magnitude estimates of saturation were a power function of colorimetric purity. In human psychophysics, it is typically found that, for prothetic continua, ratio estimation techniques, such as magnitude estimation, yield sensation scales that are not linear with such interval estimation techniques as category estimation or equisection (see Stevens \& Galanter, 1957). Possible reasons for this nonlinearity, many of which assume response biases in one or both techniques, have been discussed elsewhere (Anderson, 1974; Attneave, 1962; Curtis, Attneave, \& Harrington, 1968; Curtis \& Rule, 1972; Rule, Curtis, \& Markley, 1970; Rule, Laye, \& Curtis, 1974; Schneider, Parker, Valenti, Farrell, \& Kanow, 1978) and need not concern us here. What is important to note is that when saturation difference is scaled in both species (human and pigeon) the form of the psychophysical function is the same-namely, that saturation is linear with colorimetric purity. This suggests a strong similarity in the color mechanisms of man and pigeon despite important anatomical and physiological differences (Donovan, 1978).

\section{Theoretical Implications}

The present study provides a means of constructing a scale for a psychological attribute of a light stimulus that is based on relatively weak, nonmetric assumptions. In general, the weaker the assumptions in a scaling technique are, the more likely it is that the scale obtained via this technique is free of biases that might lead to a distorted representation of the way in which the sensory attribute is coded. To the extent that the saturation scale obtained in the present experiment is free from such biases, it describes how differences in colorimetric purity are coded by the visual system of the pigeon. Given that it is necessary only to assume that discrimination accuracy is monotonic with saturation difference, it seems reasonable to assume that the present scale provides a fairly accurate description of the way in which the pigeon codes differences in colorimetric purity.

In general, this scaling procedure would appear to be appropriate for any animal psychophysical study in which it is possible to obtain discrimination measures for pairs of stimuli. As long as these discrimination measures are monotonic with differences along a unidimensional sensory continuum, an interval scale of sensory magnitude can be constructed from their rank order. The major drawback is that it generally will take a long period of time to collect the requisite discrimination measures.

\section{REFERENCES}

Anderson, N. H. Cross-task validations of functional measurement using judgments of total magnitude. Journal of Experimental Psychology, 1974, 102, 226-233. 
Attneave, F. Perception and related areas. In S. Koch (Ed.), Psychology: A study of a science (Vol. 4). New York: McGrawHill, 1962.

Blough, D. S. spectral sensitivity in the pigeon. Journal of the Optical Society of America, 1957, 47, 827-833.

Blough, D. S. Definition and measurement in generalization research. In D. 1. Mostofsky (Ed.), Stimulus generalization. Stanford: Stanford University Press, 1965.

Blough, D., \& Blough, P. Animal psychophysics. In W. K. Honig \& J. E. R. Staddon (Eds.), Handbook of operant behavior. Englewood Cliffs, N.J: Prentice-Hall, 1977.

Blough, P. M. The pigeon's perception of saturation. Journal of the Experimental Analysis of Behavior, 1975, 24, 135-148.

BOAKES, R. A. The bisection of a brightness interval by pigeons. Journal of the Experimental Analysis of Behavior, 1969, 12, 201-209.

Carvellas, T., \& Schneider, B. Direct estimation of multidimensional tonal dissimilarity. Journal of the Acoustical Society of America, 1972, 51, 1839-1848.

Curtis, D. W., Attreave, F., \& Harrington, T. L. A test of a two-stage model of magnitude judgment. Perception \& Psychophysics, 1968, 3, 25-31.

Curtis, D. W., \& Rule, S. J. Magnitude judgments of brightness difference as a function of background reflectance. Journal of Experimental Psychology, 1972, 95, 215-222.

Donovan, W. J. Structure and function of the pigeon visual system. Physiological Psychology, 1978, 6, 403-437.

Graham, C. H., \& Hsia, Y. Saturation and the foveal achromatic interval. Journal of the Optical Society of America, 1969, 59, 993-997.

Herrnstein, R. J., \& Van Sommers, P. Method for sensory scaling with animals. Science, 1962, 135, 40-41.

InDow, T. Saturation scales for red. Vision Research, 1967, 7, $481-495$.

Indow. T., \& Stevens, S. S. Scaling of saturation and hue. Perception \& Psychophysics, 1966, 1, 253-272.

KRUSKAL, J. B. Multidimensional scaling by optimizing goodness of fit to a nonmetric hypothesis. Psychometrika, 1964, 29, 1-27.

Panek, D. W., \& Stevens, S. S. Saturation of red: A prothetic continuum. Perception \& Psychophysics, 1966, 1, 59-66.

Romeskie, M., \& Yager, D. Psychophysical studies of pigeon color vision. I: Photopic spectral sensitivity. Vision Research, 1976, 16, 501-506. (a)

Romeskie, M., \& YAger, D. Psychophysical studies of pigeon color vision. II: The spectral photochromatic interval function. Vision Research, 1976, 16, 507-512. (b)

Rule, S. J., Curtis, D. W., \& Markley, R. P. Input and output transformations from magnitude estimation. Journal of Experimental Psychology, 1970, 86, 343-349.

Rule, S. J., Laye, R. C.. \& Curtis, D. W. Magnitude judgments and difference judgments of lightness and darkness: A two-stage analysis. Journal of Experimental Psychology, 1974, 103, 1108-1114.

Schne IDer, B. Multidimensional scaling of color difference in the pigeon. Perception \& Psychophysics, 1972, 12, 373-378.

Schneider, B., Parker, S., Valenti, M., Farrell, G., \& KANOw, G. Response bias in category and magnitude estimation of difference and similarity for loudness and pitch. Journal of Experimental Psychology: Human Perception and Performance, 1978, 4, 483-496.

Shepard, R. N. Metric structures in ordinal data. Journal of Mathematical Psychology, 1966, 3, 287-315.

Stevens, S. S., \& Galanter, E. Ratio scales and category scales for a dozen perceptual continua. Journal of Experimental Psychology, 1957, 54, 377-411.

Wright, A. A. The influence of ultraviolet radiation on the pigeon's color discrimination. Journal of the Experimental Analysis of Behavior, 1972, 17, 325-337.

YAGer, D., \& Romeskie, M. On the proper control of luminance cues in pigeon color-vision experiments. Journal of the Experimental Analysis of Behavior, 1975, 23, 293-295.

Young, F. W. Nonmetric multidimensional scaling: Recovery of metric information. Psychometrika, 1970, 35, 455-473.

\section{NOTES}

1. Color temperature is not specified by the manufacturer for the 1829 bulb. However, assuming that its color temperature is within the normal range for incandescent lamps, adding an $80 \mathrm{~B}$ filter to the channel will shift the color temperature of this source above $4,000^{\circ} \mathrm{K}$.

2. This equality would not hold if the spectral coefficients of Yager and Romeskie (1975) were employed. Yager and Romeskie point out that the pigeon's retina contains two fields. The two fields have different oil droplets (red and yellow) which have different transmission spectra. Yager and Romeskie show that when the pigeon's beak is in contact with a small target $(4 \mathrm{~mm})$ on a response key, the target will project an image primarily to the red field. Consequently, when small stimuli are projected on the response key, it is advisable to use Yager and Romeskie's spectral sensitivity coefficients, which are quite different from Blough's (1957) coefficients at the shorter wavelengths. However, in the present situation, the stimulus is large enough $(17 \mathrm{~mm})$ to project to both the red and yellow fields. Consequently, Blough's (1957) coefficients were employed.

(Received for publication April 21, 1980; accepted revision received September 30,1980 .) 